$$
f_{0}(x) \geqq g(x),
$$$$
a \leqq x \leqq b .
$$

Since (43) contradicts (42), the assumption that (40) does not hold has led to a contradiction.

\title{
REFERENCES
}

1. E. F. Beckenbach, Generalized convex functions, Bull. Amer. Math. Soc. vol. 43 (1937) pp. 363-371.

2. E. F. Beckenbach and R. H. Bing, On generalized convex functions, Trans. Amer. Math. Soc. vol. 58 (1945) pp. 220-230.

3. R. Courant und D. Hilbert, Methoden der mathematischen Physik, Berlin, 1937.

4. J. L. W. V. Jensen, Sur les fonctions convexes et les inégalitês entre les valeurs moyennes, Acta Math. vol. 30 (1906) pp. 175-193.

The University of Texas

\section{NOTE ON A CERTAIN CONTINUED FRACTION}

\section{H. S. WALL}

The continued fraction

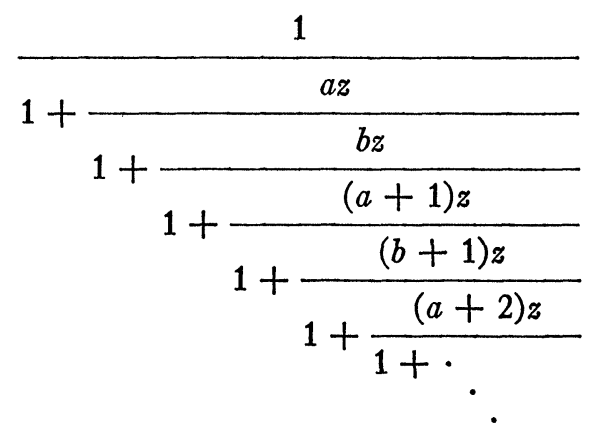

is a limiting case of the continued fraction of Gauss, and is the formal expansion of the quotient $\Omega(a, b ; z) / \Omega(a, b-1 ; z)$, where

$$
\Omega(a, b ; z)=1-a b \frac{z}{1 !}+a(a+1) b(b+1) \frac{z^{2}}{2 !}+\cdots .
$$

If $a$ and $b$ are real and positive, then it follows from the work of Stieltjes that (1) converges in the domain $Z$ exterior to the negative 
half of the real axis, and its value is an analytic function of $z$ in this domain. It is easy to show that the same holds for $a$ and $b$ any real numbers, except that the function may have poles in the domain $Z$. By means of technique which has been developed in recent years, this result may be extended to arbitrary complex $a$ and $b$. We shall first prove the following theorem.

THEOREM 1. Let $A$ and $B$ denote two arbitrary bounded regions of the complex plane. Then, there exists a number $\delta>0$, depending upon $A$ and $B$, such that the continued fraction (1) converges uniformly for $a$ in $A, b$ in $B$ and $z$ in the real interval $(0, \delta)$.

Proof. We may evidently choose $\delta>0$ sufficiently small in order that the numbers $(a+p) z,(b+p) z, p=0,1,2, \cdots$, will be in the parabolic region $|w|-R(w) \leqq 1 / 2$ for all $z$ in the interval $(0, \delta)$. The convergence then follows from the parabola theorem [3, p. 166]. ${ }^{1}$ The uniformity of the convergence follows from the fact that the approximants are uniformly bounded: their values are all in the circle with center 1 and radius 1 [4, p. 581].

TheOREM 2. Let $a$ and $b$ be arbitrary complex constants not 0 or $a$ negative integer. ${ }^{2}$ Let $G$ be any bounded closed region within the domain $Z$ defined above. The continued fraction (1) converges over $G$ except possibly at certain isolated points, and uniformly over the region obtained from $G$ by removing the interiors of small circles with centers at these points. The value of the continued fraction is an analytic function having these points as poles.

Proof. Let $A$ and $B$ of Theorem 1 be the single points $a$ and $b$, and choose the number $\delta>0$ accordingly. We may suppose that $G$ is a connected region containing the interval $(\delta / 2, \delta)$ on the interior. Let $h>0$ be chosen sufficiently small in order that $G$ will be contained within the cardioid region

$$
|w| \leqq \frac{1}{2 h^{2}}(1+\cos \theta), \quad w=|w| e^{i \theta} .
$$

Next choose $N$ so that for $n>N$ the numbers $(a+n),(b+n)$ will be in the parabolic region

$$
|w|-R(w) \leqq h^{2} / 2 .
$$

If $n>N$, the continued fraction

\footnotetext{
1 Numbers in brackets refer to the Bibliography at the end of the paper.

${ }^{2}$ If $a$ or $b$ is $\mathbf{0}$ or a negative integer, the continued fraction breaks off and is a rational fraction.
} 


$$
\frac{1}{1+\frac{(a+n) z}{1+\frac{(b+n) z}{1+\frac{(a+n+1) z}{1+\frac{(b+n+1) z}{1+}}}}}
$$

is then uniformly convergent over $G$ by the cardioid theorem [1, pp. 367-368]. It follows that (1) converges over $G$ except possibly at certain isolated points, or else diverges to the constant $\infty$. Inasmuch as it converges for $z$ in the real interval $(\delta / 2, \delta)$, the latter alternative is ruled out. The convergence is evidently uniform over the region obtained from $\mathrm{G}$ by deleting small circular neighborhoods of the aforementioned isolated points.

In order to express the analytic function represented by the continued fraction in terms of integrals, we write

$$
\begin{aligned}
\Omega(a, b ; z) & \\
& =1+\sum_{p=1}^{\infty}(-1)^{p} a(a+1) \cdots(a+p-1) b(b+1) \cdots(b+p-1) \frac{z^{p}}{p !} \\
& =\frac{1}{\Gamma(a)} \sum_{p=0}^{\infty} C_{-b, p} \Gamma(a+p) z^{p} \\
& =\frac{1}{\Gamma(a)} \sum_{p=0}^{\infty} C_{-b, p} \int_{0}^{\infty} e^{-u} u^{a+p-1} d u z^{p} \\
& =\frac{1}{\Gamma(a)} \int_{0}^{\infty} \frac{e^{-u} u^{a-1} d u}{(1+z u)^{b}} .
\end{aligned}
$$

This formal procedure suggests the possibility that (1) has the value

$$
\int_{0}^{\infty} \frac{e^{-u} u^{a-1} d u}{(1+z u)^{b}} / \int_{0}^{\infty} \frac{e^{-u} u^{a-1} d u}{(1+z u)^{b-1}}
$$

whenever these integrals converge, that is, for $z$ in $Z$ and $R(a)>0$. Now, this is known to be true for $a, b$ and $z$ real and positive [2, p. 492 ], and the extension to complex $z$ in $Z$ is immediate inasmuch as (1) and (3) are both analytic functions of $z$ over $Z$. Regarding $a$ and $b$ as variables, and using Theorem 1 , the same conclusion can be extended immediately to complex values of $a$ and $b$. 
In the special case $b=1$ :

(4)

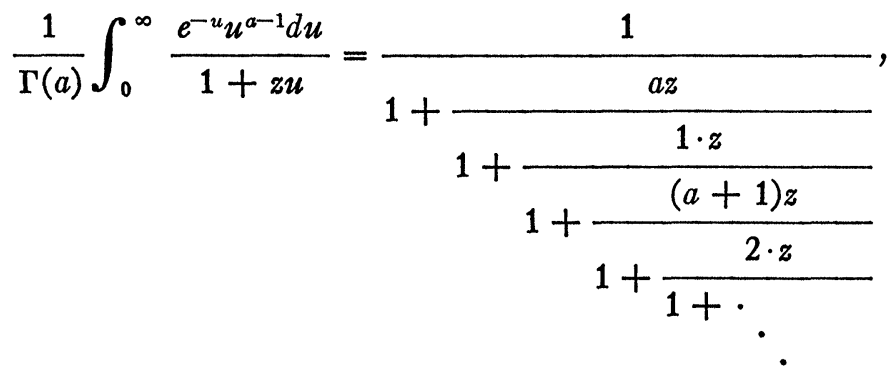

for $R(a)>0$ and $z$ in $Z$. The left-hand member can be transformed into an integral which converges for all $a$, and we get

$$
\int_{0}^{\infty} \frac{e^{-u} d u}{(z+u)^{a}}=\frac{z^{1-a}}{z+\frac{a}{1+\frac{1}{z+\frac{(a+1)}{1+\frac{2}{z+\frac{(a+2)}{1+\frac{3}{z+\cdot} \cdot}}}}}}
$$

valid for all $a$ and for $z$ in $Z$. Let $z=x$, real and positive, make the change of variable $v=u+x$ in the integral, and then replace $a$ by $1-a$. This gives

$$
\int_{x}^{\infty} e^{-v_{v}^{a-1} d v}=\frac{e^{-x} x^{a}}{x+\frac{1-a}{1+\frac{1}{x+\frac{2-a}{1+\frac{2}{x+\frac{3-a}{1+\frac{3}{x+\cdot} \cdot}}}}}}
$$

valid for all $a$ and for $x>0$. The special cases $a=0$ and $a=1 / 2$ furnish 
expansions for the integrals

$$
\int_{0}^{\sigma^{-x}} \frac{d u}{\log u} \text { and } \int_{x}^{\infty} e^{-u^{2}} d u,
$$

respectively. The latter gives immediately the expansion

$$
\begin{aligned}
& 2 \int_{0}^{x} e^{-u^{2}} d u=\pi^{1 / 2}-\frac{e^{-x^{2}}}{x+\frac{1}{2 x+\frac{2}{3+}}}, \\
& x+\frac{3}{2 x+\frac{4}{x+\cdots}}
\end{aligned}
$$

valid for $x>0$. For a discussion of these formulas, with references, see [2, pp. 296-298].

\section{BIBLIOGRAPHY}

1. J. Findlay Paydon and H. S. Wall, The continued fraction as a sequence of linear transformations, Duke Math. J. vol. 9 (1942) pp. 360-372.

2. O. Perron, Die Lehre von den Kettenbruchen, Leipzig and Berlin, 2d ed., 1929.

3. W. T. Scott and H. S. Wall, A convergence theorem for continued fractions, Trans. Amer. Math. Soc. vol. 47 (1940) pp. 155-172.

4. - Value regions for continued fractions, Bull. Amer. Math. Soc. vol. 47 (1941) pp. 580-585.

ILLINOIS INSTITUTE OF TECHNOLOGY 